\title{
A Randomized Controlled Clinical Trial Comparing 20 Gauge and 23 Gauge Vitrectomy for Patients with Macular Hole or Macular Pucker
}

\author{
Paula Scholz (D) · Philipp S. Müther · Petra Schiller • Moritz Felsch • \\ Sascha Fauser
}

Received: August 28, 2018 / Published online: November 17, 2018

(c) The Author(s) 2018

\section{ABSTRACT}

Introduction: To compare the transconjunctival sutureless 23 gauge $(G)$ pars plana vitrectomy (PPV) with 20 G PPV regarding inflammation, safety, visual outcome and patient comfort.

Methods: We included 103 patients with symptomatic macular hole or macular pucker, scheduled for vitrectomy in this prospective, randomized, controlled, mono-center clinical trial. Patients were randomized 1:1 to either $20 \mathrm{G}$ PPV $(n=51)$ or $23 \mathrm{G} \mathrm{PPV}(n=52)$. All eyes underwent standard 20G or 23G PPV with

Enhanced Digital Features To view enhanced digital features for this article go to https://doi.org/10.6084/ m9.figshare.7235525.

Electronic supplementary material The online version of this article (https://doi.org/10.1007/s12325018-0826-6) contains supplementary material, which is available to authorized users.

P. Scholz $(\varangle)$ · P. S. Müther · S. Fauser

Department of Ophthalmology, University Hospital of Cologne, Kerpener Str. 62, 50924 Cologne,

Germany

e-mail: paula.scholz@uk-koeln.de

P. Schiller · M. Felsch

Institute of Medical Statistics and Computational Biology, University Hospital of Cologne, Kerpener Str. 62, 50924 Cologne, Germany

S. Fauser

F. Hoffmann-La Roche, 4070 Basel, Switzerland membrane peeling. Primary outcome measure was change in aqueous humor flare 3 weeks after surgery compared with baseline. Secondary outcome measures were flare values 2 days and 26 weeks after surgery, subjective discomforts measured with a visual analog scale, bestcorrected visual acuity, duration of surgery, intraocular pressure (IOP) and adverse events.

Results: There was no significant difference in change of flare 3 weeks after PPV $[-1.7,95 \%$ CI ( -6.3 to 2.9 ), $p=0.466]$. Both groups showed a significant increase in flare 2 days after surgery (20G: $p<0.001,23 \mathrm{G}: p=0.002$ ), but only the $20 \mathrm{G}$ group after 3 weeks $(p=0.011)$. The gain in visual acuity after 3 weeks was higher after $23 \mathrm{G}$ PPV (4.2 95\% CI (0.4-8.0, $p=0.029)$, but without a difference after 6 months. The duration of surgery was shorter in the $23 \mathrm{G}$ group $(p<0.001)$. Patient comfort 3 weeks after surgery was greater after 23G PPV (foreign body sensation $p=0.002$; itching: $p=0.021$ ). However, the rate of complications did not differ between the groups.

Conclusion: The primary aim, showing the superiority of the $23 \mathrm{G}$ group regarding the change of flare value from baseline to 3 weeks after surgery, was not met, but the level of inflammation decreased faster after 23G PPV. Clear advantages of the 23G PPV were a lower risk of postoperative IOP elevation, a shorter surgery time, faster visual recovery and greater patient comfort in the early postoperative phase. 
Clinical Trial Registration Number: ClinicalTrials.gov NCT01969929.

Keywords: 20 Gauge; 23 Gauge; Adverse events; Macular hole; Macular pucker; Randomized controlled clinical trial; Vitrectomy

\section{INTRODUCTION}

Pars plana vitrectomy (PPV) is a standard procedure for the treatment of various vitreo-retinal diseases. The sutureless trans-conjunctival approach [1] has become the standard operating technique and replaced the conventional 20 gauge (G) PPV as the standard procedure in many centers. The smaller sclerotomy (23G or smaller versus 20G) and usually the lack of scleral and conjunctival sutures reduce surgery time and postoperative discomfort [2]. This presumably might also decrease postoperative inflammation and lead to a faster recovery [2-5]. However, Hikichi et al. reported no significant difference in anterior chamber flare values pre- and 1 week postoperatively between $20 \mathrm{G}$ and $23 \mathrm{G}$ vitrectomy in a retrospective case series [6].

The primary purpose of this study was to compare the postoperative inflammation of both surgical approaches in a prospective, randomized and blinded clinical trial. Secondary end points included surgery time, patient discomfort and visual recovery.

\section{METHODS}

This prospective, randomized and blinded clinical trial followed the tenets of the Declaration of Helsinki, was approved by the ethics committee of the University of Cologne (UKK13-247) and was registered as a clinical trial at ClinicalTrials.gov (18 October 2013, NCT01969929). Prior to study inclusion, written informed consent was obtained from all patients. For inclusion, patients had to be 18 years or older, suffer from subjective visual impairment due to a macular hole or macular pucker and be willing to return for a follow-up visit 3 weeks after surgery. Patients with prior vitreoretinal surgery or impaired vision caused by additional eye disease in the study eye were excluded.

All surgeries were performed between October 2013 and May 2015 at the Department of Ophthalmology (University Hospital Cologne, Germany) by four different surgeons, all experienced in $23 \mathrm{G}$ as well as $20 \mathrm{G}$ vitrectomy. The last follow-up was in November 2015.

\section{Randomization}

Patients were consecutively informed and screened by the trial physicians. After giving written informed consent, eligible patients were randomly assigned to one of the two treatment groups: $20 \mathrm{G}$ or $23 \mathrm{G}$ PPV (1:1 allocation). The randomization was stratified by diagnosis (macular hole vs. macular pucker) and lens status (phakic vs. pseudophakic) and implemented by sealed opaque envelopes generated by a statistician on the basis of a computergenerated randomization list (block length 4, concealed during ongoing trial).

\section{Blinding}

All patients and examiners were blinded regarding the assigned surgical procedure.

\section{Flare Measurement}

The quantitative measurement of aqueous flare was performed with the Kowa FM-500 Laser Flare-Cell Meter (version 1.0; Kowa Company, Ltd, Tokyo, Japan). The flare values were measured in both eyes $30 \mathrm{~min}$ after pupillary dilatation with $0.5 \%$ tropicamide and 5\% phenylephrine hydrochloride. A minimum of five measurements were performed in each eye and averaged. Measurements with artifacts were excluded. The laser flare values were expressed as photon counts per millisecond ( $\mathrm{pc} / \mathrm{ms})$. Flare measurements were performed at baseline (V1), 2 days after surgery (V2), 3 weeks after surgery (V3) and 6 months after surgery (V4). 


\section{Best-Corrected Visual Acuity}

Best-corrected visual acuity (BCVA) was measured with ETDRS charts at $4 \mathrm{~m}$ by a qualified, masked visual acuity examiner at V1, V3 and V4. At each visit a subjective refraction was performed prior to the visual acuity testing.

\section{Astigmatism}

The minus cylinder from the subjective refraction was used to calculate the mean astigmatism for each visit.

\section{Spherical Equivalent}

The spherical equivalent was calculated from the subjective refraction; it was defined as the sum of the spherical value and half of the cylindrical value.

\section{LOCS}

The grade of the lens opacification was estimated using the Lens Opacities Classification System III (LOCS III) at V1, V3 and V4 for all phakic patients [7]. For the analysis, the values of each parameter (nuclear color, nuclear opalescence, cortical, posterior subcapsular) were added.

\section{Patient Discomfort}

A visual analog scale with values between $0 \mathrm{~mm}$ and $100 \mathrm{~mm}$ was shown to the patients at each visit. The patients were asked to display the intensity of their discomfort regarding foreign body sensation, burning, pain, itching, photophobia and sticky feeling at the eye.

\section{Surgical Procedure}

Surgery was performed under local retro-bulbar or general anesthesia. For the PPV, a 23G onestep system or $20 \mathrm{G}$ system (EVA Phaco-vitrectomy System, DORC, Zuidland, The Netherlands) was used. A surgical posterior vitreous detachment was induced. Following this, the vitrectomy was performed. ILM peeling was accomplished after fluid-air exchange and ILM staining with $0.025 \%$ Brilliant Peel (Geuder, Heidelberg, Germany). The ILM was peeled in the entire macular area. Depending on the underlying disease, the intraocular tamponade was chosen: $\mathrm{SF}_{6}$ gas $20 \%$ (ISPAN SF${ }_{6}$ Intraocular Gas; Alcon Pharma GmbH, Freiburg, Germany) for cases with macular holes and balanced salt solution for macular pucker. After 20G PPV, sclerotomies were sutured with Vicryl 7-0, the conjunctiva with Vicyrl 9-0. After 23G PPV, scleral or conjunctival sutures were made only in cases of leaking incisions.

\section{Sample Size}

The primary end point was the change in aqueous humor flare 3 weeks after surgery (difference from flare at baseline to week 3). A 5 -point difference in the flare value (ps/ms) between groups was presumed clinically relevant. A sample size of $84(2 \times 42)$ would be required to demonstrate a difference of this size. For simplicity reasons, the calculation was based on Student's $t$ test [standard deviation (SD) in each group, derived from previous data collected by the same study group: 8 , Student's $t$ test, type 1 error rate: $5 \%$, two-sided, power $80 \%]$. Accounting for stratification by diagnosis and lens status, a total of 100 patients $(2 \times 50)$ needed to be randomized.

Calculations were performed with the G*Power 3.1.2 software (F. Faul, University of Kiel, Germany) [8].

\section{Statistical Analysis}

Efficacy analyses were based on the full analysis set (FAS). The FAS included all randomized patients who underwent eye surgery and who had a valid assessment of the baseline flare. Patients were analyzed as randomized (intention-to-treat principle). The primary end point, change of flare value from baseline to 3 weeks after surgery, was calculated using an analysis of covariance (ANCOVA) with the fixed effects group, diagnosis, lens status, surgeon and baseline flare as a covariant. Missing values were 
imputed by last observation carried forward (LOCF). The main focus was on the difference in marginal means for the change from baseline to 3 weeks. In a sensitivity analysis, the per-protocol set including all the patients who were treated according to the protocol was employed (i.e., patients for whom no major protocol deviations were documented).

Analysis of secondary end points was mainly descriptive. In addition, key secondary end points BCVA, astigmatism, spherical equivalent and LOCS were analyzed by ANCOVA, such as the primary analysis. Adverse events and aspects of patient comfort were summarized by type. Subgroup analyses regarding diagnosis (macular hole vs. macular pucker) and lens status (phakic vs. pseudophacic) were panned a posteriori. They were essentially explorative (no correction for multiple testing).

Calculations were performed with the SPSS Statistics 23 software (IBM Corp., Armonk, NY, USA).

\section{RESULTS}

One hundred three eyes of 103 patients were randomized for this study. Fifty-one patients were randomized to $20 \mathrm{G} \mathrm{PPV}$; all 51 patients were analyzed. Fifty-two were randomized to 23G PPV; 47 of those patients were analyzed for the study. Details are given in the flow diagram (Fig. 1). All baseline parameters were well balanced between the two groups (Table 1).

\section{Aqueous Flare}

The primary end point, the change of aqueous humor flare 3 weeks after surgery, did not differ between 20G and 23G PPV [20G: $3.5 \pm 9.5$, 23G: $2.3 \pm 13.2$; mean difference between groups: $-1.7,95 \%$ CI ( -6.3 to 2.9 ), $p=0.466$ ]. Nevertheless, both groups showed a significant increase in flare values (in pc/ms) 2 days after surgery. In the $20 \mathrm{G}$ group, the flare value was still significantly higher 3 weeks after surgery. At week 26, flare values were at baseline level in both groups. (20G: baseline: $9.4 \pm 6.2$, day 2: $19.1 \pm 13.0, p<0.001$, week 3: $12.9 \pm 9.9$, $p=0.011$, week $26: 10.0 \pm 5.4, p=0.490 ; 23 \mathrm{G}$ : baseline: $8.8 \pm 7.3$, day 2: $19.4 \pm 22.0$, $p=0.002$, week $3: 11.1 \pm 14.6, p=0.246$, week 26: $10.6 \pm 21.8, p=0.576$ ). See Table 2 for the evaluation of primary and key secondary end points, Tables 1 and 2 of the supplement for additional evaluation of the day 2 and week 26 parameters. Supplemental Table 3 displays the results of the evaluation of the primary and key secondary end point based on the per-protocol set.

\section{Best-Corrected Visual Acuity}

Both groups experienced a significant gain in ETDRS letters after surgery. At the week 3 visit, the increase was higher after 23G PPV (20G: $4.2 \pm 10.4,23 \mathrm{G}: 6.8 \pm 10.4, p=0.029)$, but it showed no significant difference at the 6-month visit (Table 2).

\section{Astigmatism}

The change in astigmatism was higher 3 weeks after 20G compared with 23G surgery (20G: $0.3 \pm 0.9, \quad 23 \mathrm{G}: \quad 0.1 \pm 0.6 p=0.028)$, but revealed no difference 6 months after surgery (Table 2).

\section{Spherical Equivalent}

The spherical equivalent did not show a significant change 3 weeks after surgery in either group (Table 2), but decreased significantly after 6 months in both groups (20G: $-0.1 \pm 0.6$, $p<0.001,23 \mathrm{G}:-0.1 \pm 0.9, p=0.001)$ without a relevant difference between the groups (Table 2).

\section{LOCS}

The degree of the lens opacification, assessed using the LOCS scale, increased significantly 3 weeks and 6 months after surgery in both groups, without a difference between the groups (Table 2). 


\section{CONSORT}

TRANSPARENT REPORTING of TRIALS

\section{CONSORT 2010 Flow Diagram}

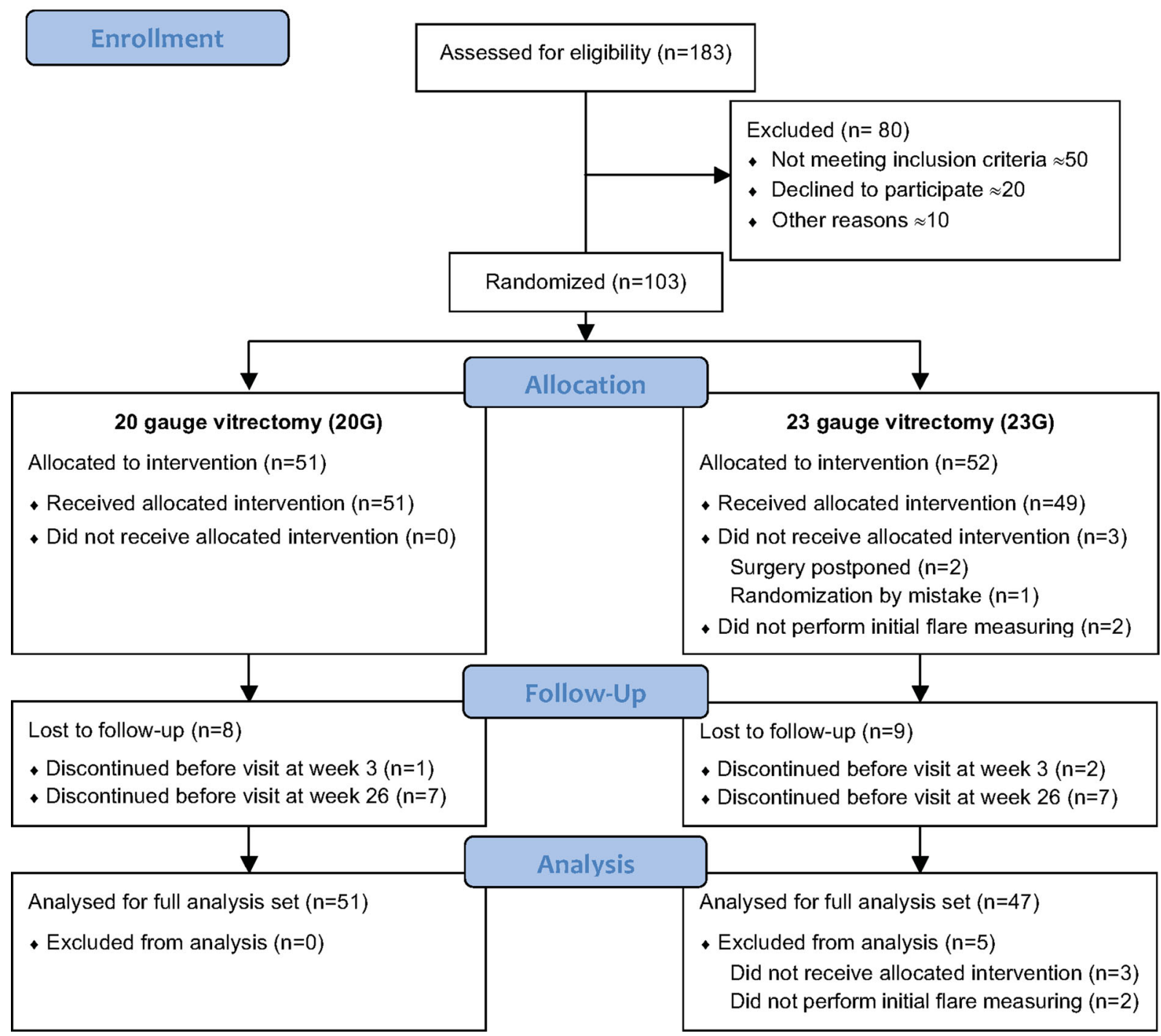

Fig. 1 Flow diagram gives an overview of the randomization process

\section{Surgery Duration}

The mean duration of surgery was shorter in the $23 \mathrm{G}$ group (20G: $29 \pm 6 \mathrm{~min}, 23 \mathrm{G}: 20 \pm 6 \mathrm{~min}$, $p<0.001)$. Details regarding the surgery duration and other intra- and postoperative outcomes are given in Table 3. 
Table 1 Preoperative characteristics of all patients (full analysis set)

\begin{tabular}{|c|c|c|c|}
\hline & $20 \mathrm{G}(n=51)$ & $23 G(n=47)$ & $P^{*}$ value \\
\hline Mean age $(S D)$, years & $68(7)$ & $68(10)$ & 0.596 \\
\hline Sex & & & 0.546 \\
\hline Male, $n(\%)$ & $24(47)$ & $19(40)$ & \\
\hline Female, $n(\%)$ & $27(53)$ & $28(60)$ & \\
\hline Eye & & & 0.225 \\
\hline Right, $n(\%)$ & $21(41)$ & $26(55)$ & \\
\hline Left, $n(\%)$ & $30(59)$ & $21(45)$ & \\
\hline \multicolumn{4}{|c|}{ Stratification by diagnosis and lens status (randomization), $n(\%)$} \\
\hline Macula hole, phakic eye & $15(29.4)$ & $12(25.5)$ & 0.983 \\
\hline Macula hole, pseudophakic eye & $8(15.7)$ & $7(14.9)$ & \\
\hline Macular Pucker, phakic eye & $18(35.3)$ & $18(38.6)$ & \\
\hline Macular Pucker, pseudophakic eye & $10(19.6)$ & $10(21.3)$ & \\
\hline Diagnosis & & & 0.686 \\
\hline Macula hole & $23(45.1)$ & $19(40.4)$ & \\
\hline Macular pucker & $28(55.0)$ & $28(59.6)$ & \\
\hline Lens status & & & 0.835 \\
\hline Phakic eye & $33(64.7)$ & $29(61.7)$ & \\
\hline Pseudophakic eye & $18(35.3)$ & $18(38.3)$ & \\
\hline Macular hole diameter $(\mu \mathrm{m})^{* *}$ & $383(160)$ & $343(132)$ & 0.519 \\
\hline
\end{tabular}

$S D$ standard deviation, $20 G 20$ gauge vitrectomy, $23 G 23$ gauge vitrectomy

${ }^{*} p$ values are from Fisher's exact test (qualitative data) or Kruskal-Wallis test (quantitative data), respectively

${ }^{* *}$ Macular hole diameter was available for all 23 patients with macular hole in the $20 \mathrm{G}$ group and all 19 patients in the $23 \mathrm{G}$ group respectively

\section{Sutures}

After 20G surgery, all conjunctival and scleral incisions were sealed with sutures as a standard feature. In the $23 \mathrm{G}$ group, in six patients the surgeon decided to make scleral sutures and in ten patients conjunctival sutures because of leaking incisions.

\section{Intraocular Pressure (IOP)}

The postoperative IOP was lower after 23G PPV (surgery day: 20G: $16.9 \pm 8.2 \mathrm{mmHg}, 23 \mathrm{G}$ : $12.5 \pm 6.2 \mathrm{mmHg}, \quad p<0.001 ;$ day $2: 20 \mathrm{G}$ :
$13.4 \pm 4.1, \quad 23 \mathrm{G}: 11.6 \pm 4.5, p=0.005)$, but matched after 3 weeks $(p=0.929)$. See Table 3 for details. The rate of postoperative hypertension was higher after 20G PPV $(p=0.033)$ but the rate of postoperative hypotension did not differ between the groups. See Table 4 .

\section{Safety and Patient Comfort}

Most analyzed parameters concerning the patient comfort showed no significant difference between 20G and 23G surgery (Table 4). A clear benefit of the 23G system was only apparent regarding the foreign body sensation 


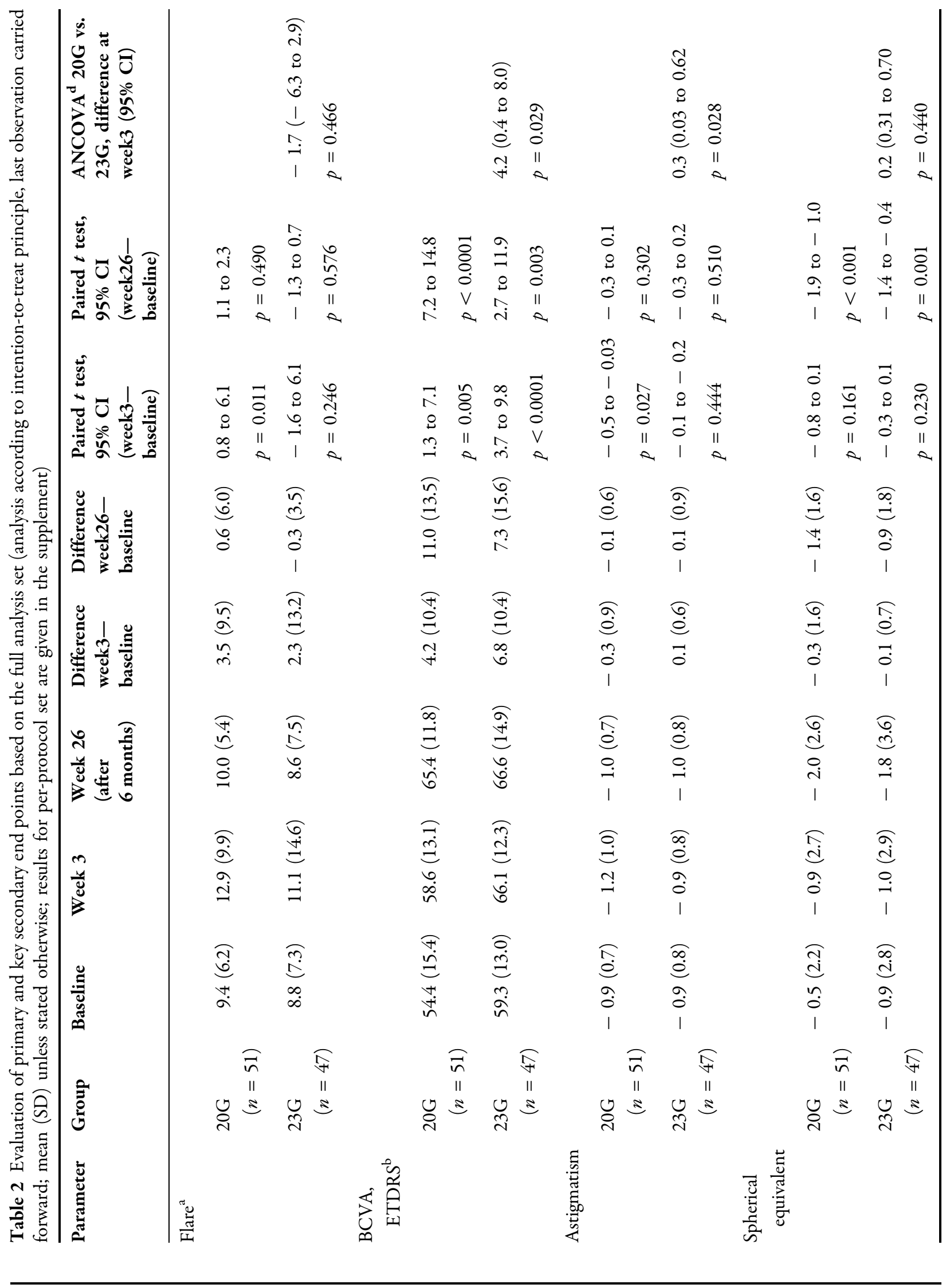




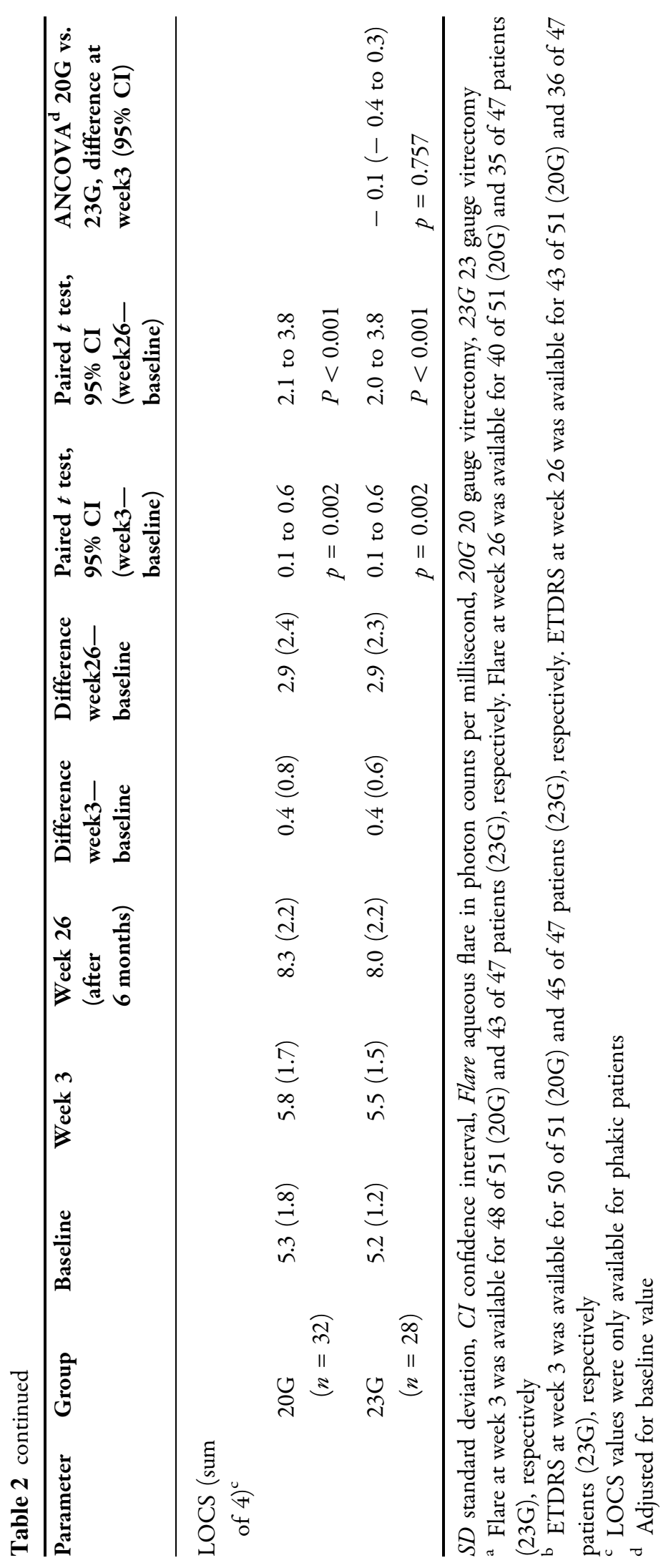


Table 3 Evaluation of intra- and postoperative outcomes/end points (mean (SD) unless stated otherwise)

\begin{tabular}{llll}
\hline Evaluation of all diagnoses & 20G $(\boldsymbol{n}=\mathbf{5 1})$ & $\mathbf{2 3 G}(\boldsymbol{n}=\mathbf{4 7})$ & $\boldsymbol{P}^{*}$ value \\
\hline Duration of surgery, min & $29(6)^{* *}$ & $20(6)^{* *}$ & $<0.001$ \\
Range & $17-47$ & $10-35$ & \\
Median (IQR) & $28(8)$ & $20(8)$ & 0.444 \\
Preoperative IOP, mmHg & $15.0(3.8)$ & $15.4(3.50)$ & $<0.001$ \\
IOP postoperative, surgery day mmHg & $16.9(8.2)^{* *}$ & $12.5(6.2)$ & 0.005 \\
IOP day 2, mmHg & $13.4(4.1)$ & $11.6(4.5)$ & 0.929 \\
IOP week 3, mmHg & $16.5(5.1)^{* *}$ & $16.7(5.5)^{* *}$ & 0.747 \\
IOP month 6, mmHg & $14.8(3.6)^{* *}$ & $15.3(3.7)^{* *}$ & 0.276 \\
Days of hospitalization & $2.1(0.6)$ & $1.9(0.5)$ & $1-3$ \\
Range & $1-3$ & $\mathbf{2 3 G}(\boldsymbol{n}=\mathbf{1 9})$ & $\boldsymbol{p}^{*}$ value \\
\hline Patients with macular hole only & $\mathbf{2 0 G}(\boldsymbol{n}=\mathbf{2 3})$ & & 0.384 \\
\hline Foramen status & $21(91)$ & $15(79)$ & $4(21)$ \\
Foramen closed & $2(9)$ & & \\
Persistent macular hole &
\end{tabular}

$I O P$ intraocular pressure, $S D$ standard deviation, $20 G 20$ gauge vitrectomy, $23 G 23$ gauge vitrectomy

${ }^{*} p$ values are from Fisher's exact test (qualitative data) or Kruskal-Wallis test (quantitative data), respectively

${ }^{* *}$ Duration of surgery was available for 50 of $51(20 \mathrm{G})$ and 47 of 47 patients (23G), respectively. ${ }^{* *}$ IOP at week 3 was available for 50 of $51(20 \mathrm{G})$ and 45 of 47 patients (23G), respectively. ${ }^{* *} \mathrm{IOP}$ at week 26 was available for 33 of 51 (20G) and 26 of 47 patients $(23 \mathrm{G})$, respectively

2 days and 3 weeks after surgery (day 2: 20G: $29 \pm 24$, 23G: $13 \pm 19, p<0.001$; week 3: 20G: $22 \pm 24,23 \mathrm{G}: 9 \pm 14, p=0.002)$ and itching 3 weeks after surgery (20G: $12 \pm 20,23 \mathrm{G}$ : $5 \pm 10, p=0.021)$. In Table 5 , all results of the visual analog scale for patient discomfort are shown.

At least one adverse event occurred in 28 of 51 patients $(55 \%)$ in the $20 \mathrm{G}$ group and in 18 from $47(38 \%)$ in the 23G group. The most common $\mathrm{AE}$ in both groups was postoperative hypertension, which appeared significantly more often after 20G PPV ( $p=0.033)$. The rate of all other adverse events did not differ between the groups. Table 4 gives an overview of the number and types of adverse events in both groups.

\section{Subgroup Analysis}

The analysis of the primary and key secondary end points for the subgroups (phakic/pseudophakic and macular hole/macular pucker) showed a statistically significant difference regarding visual acuity between the treatments for the subgroup of pseudophakic patients (9.3, 95\% CI 3.4 to $15.2 ; p=0.003$ ) as well as for the subgroup of patients with macular pucker (4.7, 95\% CI 0.3 to $9.1 ; p=0.037$ ). Pseudophakic patients as well as patients with macular pucker profited more from the 23G PPV. Regarding postoperative inflammation, none of the subgroups showed a statistically significant difference between the treatment groups. Pseudophakic patients treated with 23G PPV had a lower flare value after 3 weeks than patients treated with 20G PPV, although this 
Table 4 Tabulation of adverse events (by patient, $n$ (\%))

\begin{tabular}{lll}
\hline Category & 20G $(\boldsymbol{n}=\mathbf{5 1})$ & 23G $(\boldsymbol{n}=\mathbf{4 7})$ \\
\hline Number of AEs & 35 & 24 \\
Number of patients with at least 1 AE & $28(55)$ & $18(38)$ \\
Postoperative hypertension(30 mmHg)* & $13(25)$ & $4(9)$ \\
Iatrogenic retinal breaks & $10(20)$ & $4(9)$ \\
Steroid response (3 weeks after surgery) & $3(6)$ & $4(9)$ \\
Postoperative hypotension ( $\leq 5 \mathrm{mmHg})$ & $2(4)$ & $4(9)$ \\
Cataract surgery (within 6 months) & $3(6)$ & $2(4)$ \\
Retinal detachment & $2(4)$ & $1(2)$ \\
CNV & $0(0)$ & $1(2)$ \\
Endopthalmitis & $0(0)$ & $0(0)$
\end{tabular}

$A E$ adverse event, $C N V$ choroidal neovascularization, $20 G 20$ gauge vitrectomy, $23 G 23$ gauge vitrectomy

${ }^{*} p=0.033$ from Fisher's exact test

was not statistically significant [-3.0 95\% CI (6.2 to 0.3$) ; p=0.070]$. The flare value in the $23 \mathrm{G}$ group was still lower after 26 weeks $(11.1 \pm 11.3)$ compared with $20 \mathrm{G}(12.2 \pm 6.6)$, (Figs. 2, 3, Supplemental Table 4).

\section{DISCUSSION}

The transconjunctival 23G PPV has become a standard procedure in many centers. Possible advantages could be less postoperative inflammation, a shorter duration of surgery and greater patient comfort due to smaller incisions and a sutureless approach [2-4, 9]. However, the data published so far are inconsistent, and most studies have major limitations such as lack of randomization, retrospective study design, lack of blinding of patients and non-uniform surgery indications.

In this randomized and blinded clinical trial, postoperative inflammation, visual outcome and patient comfort were compared between $20 \mathrm{G}$ versus $23 \mathrm{G}$ PPV. There was no statistically significant difference in aqueous humor flare values between the two groups 3 weeks after surgery, and therefore the primary end point was not met. However, inflammation decreased faster in the $23 \mathrm{G}$ group. Also, the subgroup analysis showed that pseudophakic patients favored 23G PPV over 20G PPV, although the difference was not significant (Fig. 2).

Hence, it appears that the inflammation caused by the actual surgery is more or less similar in 20G and 23G PPV, but regarding the $20 \mathrm{G} \mathrm{PPV}$, there seems to be a remaining inflammation stimulus after surgery, which could be responsible for the extended increase in flare values beyond the week 3 visit. The most probable explanation for the slower decrease in inflammation after 20G PPV is the sutures used in 20G PPV to seal the incisions. Although the used material (vicryl) is usually well tolerated, it is still a foreign body that can induce inflammation in the eye. Also, the larger incision itself could be responsible for the slower decrease in postoperative inflammation after 20G PPV.

So, even if there might be a scant but quantifiable advantage for the 23G PPV regarding the postoperative inflammation, since the effect is only short term, it is highly questionable if this leads to any relevant benefit for the daily clinical practice.

Rizzo et al. [10] reported a lower postoperative inflammation 1 day and 1 week after sutureless small-incision PPV, but they 
Table 5 Patient comfort [measured with a visual analog scale; mean (SD)]

\begin{tabular}{|c|c|c|c|}
\hline Item & Time & $20 \mathrm{G}(n=51 \dagger)$ & $23 G(n=47 \dagger)$ \\
\hline \multirow[t]{4}{*}{ Foreign body sensation } & Baseline & $13(21)$ & $9(21)$ \\
\hline & Day $2^{*}$ & $29(24)$ & $13(19)$ \\
\hline & Week $3^{* *}$ & $22(24)$ & $9(14)$ \\
\hline & Month 6 & $7(19)$ & $6(16)$ \\
\hline \multirow[t]{4}{*}{ Pain } & Baseline & $4(13)$ & $2(7)$ \\
\hline & Day $2^{* * *}$ & $10(18)$ & $6(10)$ \\
\hline & Week 3 & $6(11)$ & $4(14)$ \\
\hline & Month 6 & $4(15)$ & $2(10)$ \\
\hline \multirow[t]{4}{*}{ Burning } & Baseline & $8(15)$ & $7(15)$ \\
\hline & Day 2 & $15(20)$ & $8(16)$ \\
\hline & Week 3 & $10(19)$ & $8(17)$ \\
\hline & Month 6 & $7(17)$ & $5(17)$ \\
\hline \multirow[t]{4}{*}{ Itching } & Baseline & $14(18)$ & $9(17)$ \\
\hline & Day 2 & $6(11)$ & $6(12)$ \\
\hline & Week $3^{+}$ & $12(20)$ & $5(10)$ \\
\hline & Month 6 & $6(17)$ & $3(7)$ \\
\hline \multirow[t]{4}{*}{ Sticky feeling at the eye } & Baseline & $8(15)$ & $7(15)$ \\
\hline & Day 2 & $24(26)$ & $15(20)$ \\
\hline & Week 3 & $13(21)$ & $8(14)$ \\
\hline & Month 6 & $8(18)$ & $4(10)$ \\
\hline \multirow[t]{4}{*}{ Photophobia } & Baseline & $24(28)$ & $30(32)$ \\
\hline & Day 2 & $24(28)$ & $23(26)$ \\
\hline & Week 3 & $23(27)$ & $27(30)$ \\
\hline & Month $6^{++}$ & $15(25)$ & $29(37)$ \\
\hline
\end{tabular}

SD standard deviation, $20 G 20$ gauge vitrectomy, $23 G 23$ gauge vitrectomy

a Completed forms were missing at day 2 for 1 patient (20G), at week 3 for 4 patients ( 1 in $20 \mathrm{G}$ and 3 in $23 \mathrm{G}$ ) and at week 26 for 19 patients ( 8 in $20 \mathrm{G}$ and 11 in $23 \mathrm{G}) .{ }^{*} p<0.001,{ }^{* *} p=0.002,{ }^{* * *} p=0.06,{ }^{+} p=0.021,{ }^{++} p=0.050$ (derived from $t$ test)

compared 25G and 20G PPV, using a subjective semiquantitative score to classify the degree of postoperative inflammation. Therefore, the results are not quite comparable to our findings. Hikichi et al. also reported increased flare values 1 week after 20G and 23G surgery in a retrospective case series, but they did not find a difference between the two groups [6]. Unfortunately, they did not publish any flare data measured later than 1 week after surgery.

In addition to the aqueous flare, we also looked into visual acuity improvement after surgery. This was faster after 23G PPV, but 


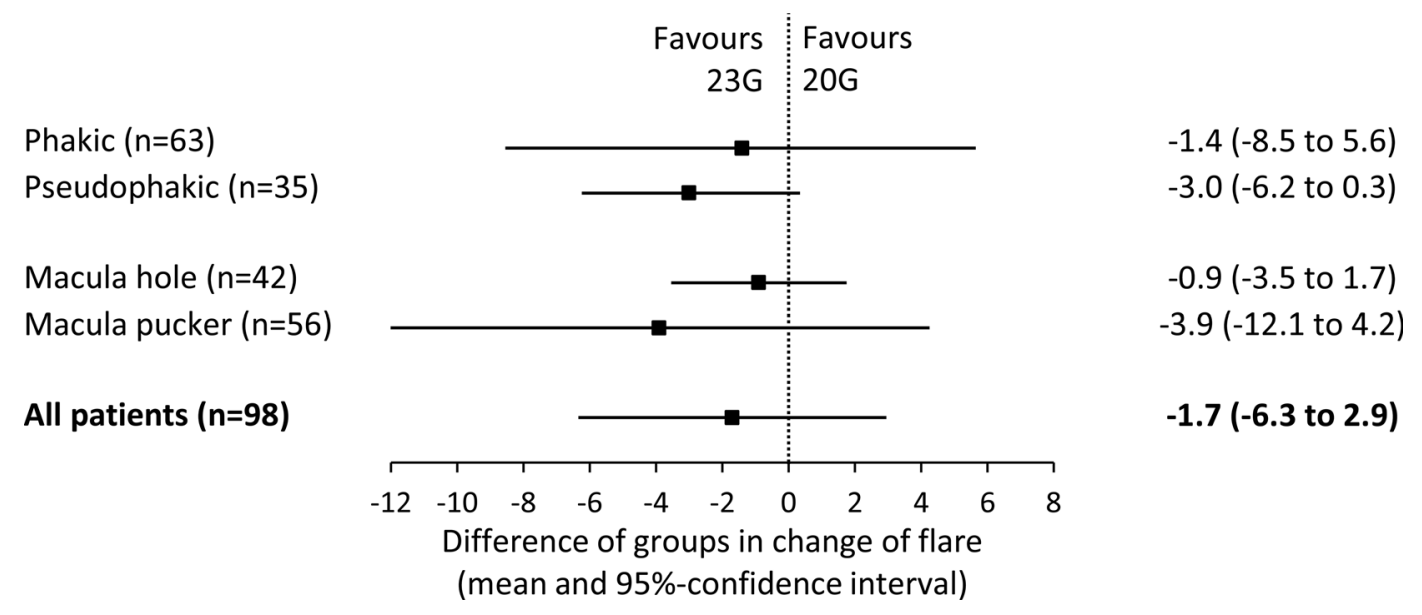

Fig. 2 Forest plots for the subgroup analysis regarding the change in flare values from baseline to week 3. 23G vitrectomy is favored in all subgroups. $B C V A$ best corrected visual acuity, flare aqueous flare in photon

counts per millisecond; $20 G 20$ gauge vitrectomy, $23 G 23$ gauge vitrectomy
Phakic $(n=63)$

Pseudophakic $(n=35)$

Macula hole $(n=42)$

Macula pucker $(n=56)$

All patients $(n=98)$

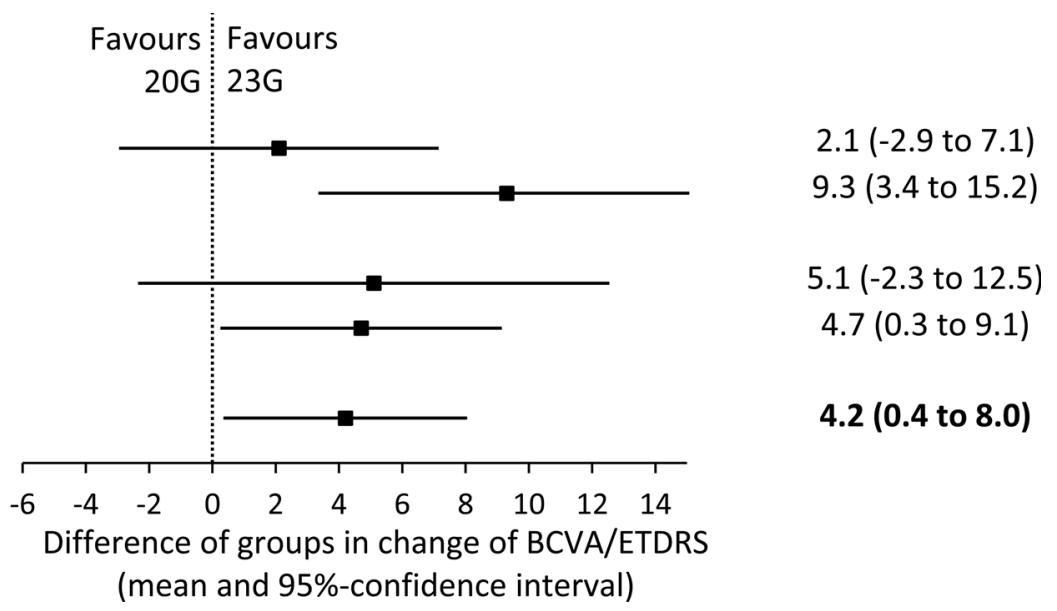

Fig. 3 Forest plots for the subgroup analysis regarding the change in EDTRS letters from baseline to week 3. 23G vitrectomy is favored in all subgroups. BCVA best corrected visual acuity, ETDRS early treatment diabetic

without a better visual acuity at the last visit after 26 weeks.

This finding is in line with data published by Hikichi et al. [6] and Narayanan et al. [11]. Most authors ascribed the faster visual recovery to the lower early postoperative corneal astigmatism after small-incision surgery, which was reported in different studies $[6,12,13]$ and also confirmed by our work. Furthermore, the increased anterior chamber inflammation found in the retinopathy study, $20 G 20$ gauge vitrectomy, $23 G 23$ gauge vitrectomy

20G group 3 weeks after surgery could be an additional reason for the slower visual recovery.

A clear advantage of the small-incision PPV is the shorter surgery time. Similar to other studies $[3,4], 23 \mathrm{G}$ PPV shortened the surgery duration in our cohort significantly. The reduction of the surgery time by a mean of 9 min per patient can likely compensate for the higher costs of the 23G PPV set at least partially by lowering the 
costs of surgery staff and the time in the surgery room.

Another potentially relevant advantage of the $23 \mathrm{G}$ technique is the better postoperative patient comfort and less postoperative pain. A faster recovery is not only a matter of personal comfort but could also be an economic factor regarding the number of sick days after surgery. However, postoperative pain values were very low in both groups and did not differ significantly between the groups. Nevertheless, patients in the $23 \mathrm{G}$ group had significantly less foreign body sensation, eye burning and sticky feeling in the early postoperative phase, which could be explained by the lack of sutures after $23 \mathrm{G}$ PPV. However, the greater postoperative comfort did not lead to a shorter duration of hospitalization in our cohort. Likewise, Mentens et al. did not find a difference regarding the number of sick days after surgery between $20 \mathrm{G}$ and 23G PPV despite the greater patient comfort after sutureless PPV in their study [2].

With the introduction of the small-incision surgery, surgeons not only hoped for an easy and fast-to-perform technique, but also for fewer complications, since the technique seems to be gentler and less invasive.

A postoperative IOP rise is a common finding after PPV [14]. Gosse et al. found a higher rate of IOP elevation after 20G compared with 23G PPV in a retrospective case series [15].

In our study, we also found a statistically significantly higher IOP after 20G PPV. Additionally, the risk of postoperative IOP elevation $\geq 30 \mathrm{mmHg}$ was significantly higher after 20G PPV. Although the postoperative hypertension is usually transient, the management normally includes local or even systemic IOPlowering medication and more frequent examination visits. Even if the hypertension is treated successfully after detection, also a transient hypertensive episode can potentially lead to long-term damage. The risk might be especially high for patients after outpatient surgery when the interval between the appearance of the hypertension and detection by the ophthalmologist can be prolonged.

Furthermore, an idea could be that a lower early postoperative pressure, as found after 23G $\mathrm{PPV}$, reduces the risk of long-term glaucoma development. However, so far, whether PPV increases the risk of glaucoma [16] and if the risk is lower after 23G surgery have not been clarified yet. In our cohort, all patients with postoperative hypertension could be temporarily treated with local or systemic anti-glaucoma drugs without long-term complications during the 6-month follow-up period. The lower risk ofor postoperative hypertension after 23G PPV could be accompanied by a higher rate of postoperative hypotension as reported by Haas et al. We could not see this effect in our study; the occurrence of postoperative hypotension did not differ between the two surgery techniques.

The most feared complications after PPV are postoperative endophthalmitis and retinal detachment. Like other studies $[4,6]$, we could not detect any difference in the appearance of those complications. Additionally, the rate of patients with any AE and the overall number of AEs did not differ between the groups.

A typical side effect of PPV in phakic patients is cataract formation $[17,18]$. We also saw a low but significant increase in LOCS values as early as week 3 and a much more distinct increase 6 months after surgery in our patients. We also observed a shift of the spherical equivalent toward minus in both groups during the observational period. This is most likely caused by a myopic shift due to the cataract formation. It is conceivable that the shorter surgery duration in the $23 \mathrm{G}$ group leads to a lower stimulation of cataract formation. However, we did not find any difference in the degree of cataract development between the two groups. Besides, there was also no difference in the number of patients with the need for cataract surgery within the first 6 months after surgery.

Not really a complication, but nevertheless an important surgical outcome, is the closure rate of macular holes. We found no significant difference in the closure rate of macular holes between the two groups. Also Krishnan et al. did not find different closure rates after 20G and 23G PPV in a retrospective case series [5].

Limitations of our study are the lack of evaluation visits between week 3 and week 26 . Therefore, we are not able to narrow down the time frame with increased flare values for the 
different groups. Additionally, the cohort is too small to perform convincing statistical analysis for the subgroups (macular hole, macular pucker with subdivision in phakic and pseudophakic). Our subgroup analyses show that pseudophakic patients as well as patients with macular pucker profit more from the 23G PPV regarding visual outcome (Figs. 2, 3). With respect to postoperative inflammation, none of the subgroups showed a statistically significant difference between the treatment groups. Due to small subgroups and therefore wide confidence intervals, only a trend toward lower flare values after $23 \mathrm{G}$ PPV could be shown in pseudophakic patients.

\section{CONCLUSIONS}

In conclusion, the primary aim of this trial, the superiority of the 23G PPV regarding the change of flare value from baseline to 3 weeks after surgery, could not be shown. However, the level of inflammation decreases faster after 23G PPV. Nevertheless, clear advantages of the 23G PPV are a lower risk for postoperative IOP elevation, a shorter surgery time, faster visual recovery and a greater patient comfort in the early postoperative phase.

\section{ACKNOWLEDGEMENTS}

We thank the participants of the study.

Funding. No funding or sponsorship was received for this study or publication of this article. The article processing charges were funded by the authors. The authors have no proprietary or commercial interest in any materials discussed in this article.

Authorship. All named authors meet the International Committee of Medical Journal Editors (ICMJE) criteria for authorship for this article, take responsibility for the integrity of the work as a whole and have given their approval for this version to be published.
Authorship Contributions. SF and PM conceived the idea. SF, PM, PS and MF designed the experiment. PS and PS analyzed the data. PS wrote the manuscript in accordance with discussions with SF, PM and PS. All authors reviewed the manuscript.

Disclosures. All authors (Paula Scholz, Philipp Müther, Petra Schiller, Moritzs Felsch and Sascha Fauser) have nothing to disclose.

Compliance with Ethics Guidelines. This clinical trial followed the tenets of the Declaration of Helsinki of 1964, as revised in 2013, concerning human and animal rights; additionally, Springer's policy concerning informed consent has been followed. It was approved by the ethics committee of the University of Cologne (UKK-13-247) and registered as a clinical trial at ClinicalTrials.gov (18 October 2013, NCT01969929). All procedures performed in studies involving human participants were in accordance with the ethical standards of the institutional national research committee and with the 1964 Helsinki Declaration and its later amendments or comparable ethical standards. Informed consent was obtained from all individual participants included in the study.

Data Availability. The data sets generated during and analyzed during the current study are not publicly available because of further analyses but are available from the corresponding author on reasonable request.

Data Availability-Protocol. The full protocol of this study is available from the corresponding author on reasonable request.

Open Access. This article is distributed under the terms of the Creative Commons Attribution-NonCommercial 4.0 International License (http://creativecommons.org/licenses/ by-nc/4.0/), which permits any noncommercial use, distribution, and reproduction in any medium, provided you give appropriate credit to the original author(s) and the source, provide a link to the Creative Commons license, and indicate if changes were made. 


\section{REFERENCES}

1. Eckardt C. Transconjunctival sutureless 23-gauge vitrectomy. Retina. 2005;25(2):208-11.

2. Mentens R, Stalmans P. Comparison of postoperative comfort in 20 gauge versus 23 gauge pars plana vitrectomy. Bull Soc Belge Ophtalmol. 2009;311:5-10.

3. Misra A, Ho-yen G, Burton R. 23-Gauge sutureless vitrectomy and 20-gauge vitrectomy: a case series comparison. Eye. 2009;23(5):1187.

4. Haas A, Seidel G, Steinbrugger I, Maier R, GasserSteiner V, Wedrich A, et al. Twenty-three-gauge and 20-gauge vitrectomy in epiretinal membrane surgery. Retina. 2010;30(1):112-6.

5. Krishnan R, Tossounis C, Yang YF. 20-Gauge and 23-gauge phacovitrectomy for idiopathic macular holes: comparison of complications and long-term outcomes. Eye. 2012;27(1):72.

6. Hikichi T, Matsumoto N, Ohtsuka H, Higuchi M, Matsushita T, Ariga $\mathrm{H}$, et al. Comparison of oneyear outcomes between 23-and 20-gauge vitrectomy for preretinal membrane. Am J Ophthalmol. 2009;147(4):639-43 e1.

7. Chylack LT, Wolfe JK, Singer DM, Leske MC, Bullimore MA, Bailey IL, et al. The lens opacities classification system III. Arch Ophthalmol. 1993;111(6):831-6.

8. Faul F, Erdfelder E, Lang A-G, Buchner A. G* Power 3: a flexible statistical power analysis program for the social, behavioral, and biomedical sciences. Behav Res Methods. 2007;39(2):175-91.

9. Krishnan R, Tossounis C, Yang YF. 20-gauge and 23-gauge phacovitrectomy for idiopathic macular holes: comparison of complications and long-term outcomes. Eye. 2013;27(1):72-7.

10. Rizzo S, Genovesi-Ebert F, Murri S, Belting C, Vento A, Cresti F, et al. 25-gauge, sutureless vitrectomy and standard 20-gauge pars plana vitrectomy in idiopathic epiretinal membrane surgery: a comparative pilot study. Graefe's Arch Clin Exp Ophthalmol. 2006;244(4):472-9. https://doi.org/10.1007/ s00417-005-0173-6.

11. Narayanan R, Sinha A, Reddy RK, Krishnaiah S, Kuppermann BD. Faster visual recovery after 23-gauge vitrectomy compared with 20-gauge vitrectomy. Retina. 2010;30(9):1511-4.

12. Okamoto F, Okamoto C, Sakata N, Hiratsuka K, Yamane N, Hiraoka T, et al. Changes in corneal topography after 25-gauge transconjunctival sutureless vitrectomy versus after 20-gauge standard vitrectomy. 2007;114(12):2138-41.

Ophthalmology.

13. Kadonosono K, Yamakawa T, Uchio E, Yanagi Y, Tamaki Y, Araie M. Comparison of visual function after epiretinal membrane removal by 20 -gauge and 25-gauge vitrectomy. Am J Ophthalmol. 2006;142(3):513-5.

14. Desai UR, Alhalel AA, Schiffman RM, Campen TJ, Sundar G, Muhich A. Intraocular pressure elevation after simple pars plana vitrectomy. Ophthalmology. 1997;104(5):781-6.

15. Gosse E, Newsom R, Hall P, Lochhead J. Changes in day 1 post-operative intraocular pressure following sutureless 23-gauge and conventional 20-gauge pars plana vitrectomy. Open Ophthalmol J. 2013;7:42.

16. Thompson JT. Does vitrectomy increase the risk of glaucoma? LWW. 2011.

17. Melberg NS, Thomas MA. Nuclear sclerotic cataract after vitrectomy in patients younger than 50 years of age. Ophthalmology. 1995;102(10):1466-71.

18. Cherfan GM, Michels RG, de Bustros S, Enger C, Glaser BM. Nuclear sclerotic cataract after vitrectomy for idiopathic epiretinal membranes causing macular pucker. Am J Ophthalmol. 1991;111(4):434-8. 